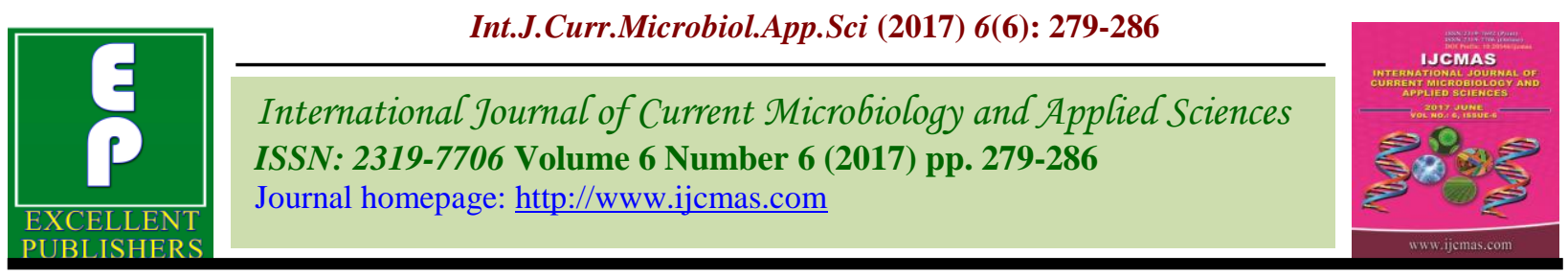

Original Research Article

https://doi.org/10.20546/ijcmas.2017.606.034

\title{
Isolation and Antibiotic Sensitivity Pattern of Extended Spectrum Beta Lactamases (ESBL) Producing Escherichia coli Isolated from Urinary Tract Infection
}

\author{
Ravindranath Gangane and Javeria Firdous* \\ Department of Microbiology, Mahadevappa Rampure Medical College, \\ Kalaburagi, Karnataka, India \\ *Corresponding author
}

A B S T R A C T

Escherichia coli is the most common organism causing urinary tract infection (UTI). This organism has the ability to produce Extended Spectrum Beta

Keywords

E. coli,

UTI, ESBL,

Kirby Bauer disk

diffusion method,

CLSI.

Article Info

Accepted:

04 May 2017

Available Online:

10 June 2017
Lactamases (ESBLs), which confer multiple drug resistance making urinary tract infection difficult to treat. So treatment of UTI requires constant updating of the antibiotic sensitivity profile. Objectives of this study were to detect prevalence of ESBL production among $E$. coli isolates causing urinary tract infection and to detect their antibiotic susceptibility pattern. A total of 400 consecutive, nonrepetitive E. coli isolates were studied. Antimicrobial susceptibility test was performed using Kirby Bauer disk diffusion method. ESBL detection was done for all isolates according to latest CLSI criteria. Out of $400 \mathrm{E}$. coli isolate, 244(61\%) were ESBL producers and 156(39\%) were Non ESBL producers. The isolates were highly susceptible to imipenem (100\%) and Piperacillin/Tazobactum $(88.1 \%)$ and were least susceptible to Ampicillin (100\%) and Cotrimoxazole $(89.7 \%)$. This study demonstrate the importance of regular review of empirical antibiotic therapy for UTI in view of the evolving resistance of ESBL producing E. coli to commonly used antimicrobial agents.

\section{Introduction}

Extended spectrum beta lactamases (ESBLs) producing bacteria are typically resistant to penicillins, first and second generation cephalosporins as well as the third generation oxyiminocephalosporins (e.g., Ceftazidime, Ceftriaxone) and Monobactam (Aztreonam) except cephamycins and carbapenems (Rawat et al., 2010).

The persistent exposure of the bacterial strains to a multitude of $\beta$-lactams has induced a dynamic and continuous production and mutation of $\beta$-lactamases in the bacteria. ESBL enzymes are plasmid borne and they have evolved from point mutations which altered the configuration of the active site of the original and long known $\beta$-lactamases, which have been designated as TEM-1, TEM2 and SHV-1 (Nathisuwan et al., 2001). The resistance to newer $\beta$-lactams which are a result of these $\beta$-lactamases has emerged quickly. These enzymes are commonly produced by many members of Enterobacteriaceae, especially E. coli and $K$. 
pneumoniae. First isolated in 1983 in Germany, ESBLs spread rapidly to Europe, United States and Asia and are now found all over the world (Suganya et al., 2014). Since ESBL positive isolates show false susceptibility to extended spectrum cephalosporins in standard disk diffusion tests (Kumar et al., 2006). It is difficult to reliably detect ESBL production by the routine disk diffusion techniques. Specific detection methods such as double disk potentiation methods recommended by CLSI (2016) have to be adopted. ESBLs are inhibited by ßlactamase inhibitors like clavulanic acid, sulbactam and tazobactam and this property of specific inhibition can be utilized for the detection and confirmation of ESBLs.

It is estimated that there are about 150 million urinary tract infections per annum worldwide (Stamm et al., 2001). Escherichia coli is the most common organism causing urinary tract infection (UTI). This organism has the ability to produce ESBLs, which confer multiple drug resistance making urinary tract infection difficult to treat (Kariuki et al., 2007).

Delay or failure in identifying and reporting ESBL production contributes to their uncontrolled spread. Infections with ESBL are associated with prolonged hospital stay, increased morbidity, mortality, and health care costs. Many clinical laboratories are still not aware of the importance of screening for ESBL-producing E. coli.

A heightened awareness of these organisms by clinicians and enhanced testing by laboratories is the need of the hour. Knowledge of antibiotic resistance pattern will help in the appropriate and judicious antibiotic use. The main obje ctives of this study includes to detect prevalence of ESBL production among E. coli isolates causing urinary tract infection and also to detect their antibiotic susceptibility pattern.

\section{Materials and Methods}

The patients admitted and / or attending the outpatient department in Basaveshwara teaching and general hospital, Kalaburagi, Karnataka from September 2016 to January 2017 with signs and symptoms suggestive of urinary tract infection were included in the study. The study was approved by the institutional ethics committee. Informed consent was taken from all the patients. A total of 400 consecutive, non-repetitive $E$. coli isolates were studied during this period.

\section{Isolation of pathogens}

Urine specimens were inoculated onto Blood agar, MacConkey agar and CLED agar by using standard techniques. Plates were incubated at $37^{\circ} \mathrm{C}$ for overnight before the plates were inspected for growth. Gram's staining was performed (Cheesbrough, 1989).

\section{Identification of isolates}

Identification of all isolates was done on the basis of routine biochemical tests i.e., Gram staining, catalase test, oxidase test, motility,indole production, methyl red test, vogesproskauer test, citrate utilization test, nitrate reduction test, triple sugar iron test, urease production, sugar fermentation test and amino acid decarboxylation tests using standard techniques (Baird, 2014).

\section{Antimicrobial susceptibility test}

This was performed using Kirby Bauer disk diffusion method. Following antibiotic disks were used: Amikacin $(30 \mu \mathrm{g})$, Gentamicin (10 $\mu \mathrm{g})$, Amoxicillin/Clavulanate $(20 / 10 \mu \mathrm{g})$, Ceftazidime $(30 \mu \mathrm{g})$, Cefepime $(30 \mu \mathrm{g})$, Cefuroxime $(30 \mu \mathrm{g})$, Ciprofloxacin $(5 \mu \mathrm{g})$, Cotrimoxazole $(1.25 / 23.75 \mu \mathrm{g})$, Nalidixic acid $(30 \mu \mathrm{g})$, Nitrofurantoin $(300 \mu \mathrm{g})$, Norfloxacin (10 $\mu \mathrm{g})$, Piperacillin/ Tazobactum (100/10 $\mu \mathrm{g})$, Imipenem $(10 \mu \mathrm{g})$. 
The disk were obtained from high media laboratories. The diameter of zone of inhibition was measured and interpreted according to CLSI guidelines (2016).

\section{Detection of ESBL}

ESBL detection was done for all isolates according to latest CLSI criteria.

\section{Screening test}

According to latest CLSI guidelines, zone diameter of $E$. coli strain for ceftazidime $<22 \mathrm{~mm}$ and for cefotaxime $<$ $21 \mathrm{~mm}$ is presumptively taken to indicate ESBL production.

\section{Confirmatory test}

As per CLSI guidelines, ESBLs were confirmed by placing disk of cefotaxime and ceftazidime at a distance of $20 \mathrm{~mm}$ from a disk of cefotaxime/clavulanate $(30 / 10 \mu \mathrm{g})$ and ceftazidime/clavulanate $(30 / 10 \mu \mathrm{g})$ respectively on a lawn culture of test strain (0.5 McFarland inoculum size) on Mueller-Hinton agar. After overnight incubation at $37^{\circ} \mathrm{C}$, ESBL productionwas confirmed if there was $\geq 5 \mathrm{~mm}$ increase in zone diameter for either antimicrobial agent tested in combination with clavulanate versus its zone when tested alone.

\section{Results and Discussion}

Table 1 shows the number and percentage of ESBL and Non ESBL producing E. coli isolates. Out of $400 \mathrm{E}$. coli isolate, 244(61\%) are ESBL producers and 156(39\%) are Non ESBL producers. Graph 1 shows ESBL producers among E. coli isolates in UTI. Table 2 shows the number and percentage of ESBL producing $E$. coli isolates in males and females. Out of 244 ESBL producing $E$. coli isolates, $90(36.89 \%)$ were found in males and
$154(63.11 \%)$ in females. Graph 2 shows the gender distribution of ESBL producing $E$. coli isolates.

Table 3 and Graph 3 shows the antibiotic susceptibility pattern of ESBL producing $E$. coli isolates from UTI. All ESBL producers were resistant to Ampicillin. 93.8\% were resistant to Cotrimoxazole, $89.7 \%$ were resistant to Nalidixic acid, $88.1 \%$ were resistant to Gentamicin, $84 \%$ were resistant to Amoxicillin/Clavulanate, $82 \%$ were resistant to Ciprofloxacin, $73.7 \%$ were resistant to Ceftazidine, $69 \%$ were resistant to Norfloxacin, $59.8 \%$ were resistant to Amikacin, 27\% were resistant to Nitrofurantoin, $11.9 \%$ were resistant to Piperacillin/Tazobactum and all the ESBL producers were sensitive to Imipenem.

Urinary tract infections are the most common bacterial infection (Foxman, 2002). Escherichia coli is the most common organism causing urinary tract infection (UTI). Extended spectrum beta - lactamases (ESBLs) are on the rise in hospital settings across the globe (Sulochana et al., 2013). The antimicrobial resistance patterns of organisms-causing UTI are changing over the years, including resistance due to ESBL producing pathogens. Correct identification of ESBL producing organisms in due time is necessary not only for optimal patient management but also for immediate institution of appropriate infection control measures to prevent the spread of these organism (Sasirekha, 2013). This study was a small step towards the same.

In the present study it was observed that $61 \%$ of $E$. coli isolates were ESBL producers. Studies done in other parts of the country have shown an incidence between $21 \%$ and $82 \%$ (Table 4). The wide variation in prevalence may be due to differences in the risk factors, the extent of antibiotic use, and 
the selection of organisms for study. The high incidence in our center was probably due to the fact that it is a tertiary care center with high usage of antibiotics, especially 3rd generation cephalosporins.

In our study prevalence of ESBL producing E. coli was found to be $61 \%$. This is in correlation with other studies such as Mahesh et al., (2010) and Chaudhary et al., (2013) who reported $56.2 \%$ and $54.5 \%$ ESBL production in E. coli isolates respectively. However our findings are in contrast with other studies conducted by Datta et al., (2014) Dugal et al., (2013) DMBT Dissanayake et al., (2012) and Singh et al., (2016) who reported $21.4 \%, 24.4 \%, 29 \%$ and $82.6 \%$ ESBL producing $E$. coli isolates respectively.

Gender wise distribution of ESBL revealed a female preponderance $(63.11 \%)$ over males $(36.89 \%)$. This may be due to the fact that UTI is more common in females, principally owing to anatomic and physical Factors. This is similar to studies done by Sasirekha et al., (2013) and Rajan et al., (2012).

Table.1 ESBL producers among E. coli isolates

\begin{tabular}{|l|l|l|l|l|}
\hline Total number of & \multicolumn{3}{|l|}{ ESBL producers } & Non ESBL producers \\
\cline { 2 - 5 } E.coli isolates & Number & $\%$ & Number & $\%$ \\
\hline 400 & 244 & 61 & 156 & 39 \\
\hline
\end{tabular}

Table.2 Gender distribution of ESBL positive E. coli isolates

\begin{tabular}{|l|l|l|l|l|}
\hline Total number of & \multicolumn{2}{|l|}{ ESBL producers in males } & \multicolumn{2}{l|}{ ESBL producers in females } \\
\cline { 2 - 5 } ESBL isolates & Number & $\%$ & Number & $\%$ \\
\hline 244 & 90 & $36.89 \%$ & 154 & $63.11 \%$ \\
\hline
\end{tabular}

Table.3 Antibiotic susceptibility pattern of ESBL producing E. coli isolates from UTI

\begin{tabular}{|l|l|l|l|}
\hline Antibiotics & Sensitive & Intermediate & Resistant \\
\hline Ampicillin & 0 & 0 & $244(100 \%)$ \\
\hline Ciprofloxacin & $44(18 \%)$ & 0 & $200(82 \%)$ \\
\hline Cotrimoxazole & $11(4.5 \%)$ & $4(1.7 \%)$ & $229(93.8 \%)$ \\
\hline Amikacin & $59(24.2 \%)$ & $39(16 \%)$ & $146(59.8 \%)$ \\
\hline Gentamicin & $15(6.2 \%)$ & $14(5.7 \%)$ & $215(88.1 \%)$ \\
\hline Amoxicillin/Clavulanate & $39(16 \%)$ & 0 & $205(84 \%)$ \\
\hline Ceftazidime & $55(22.6 \%)$ & $9(3.7 \%)$ & $180(73.7 \%)$ \\
\hline Nalidixic acid & $25(10.3 \%)$ & 0 & $219(89.7 \%)$ \\
\hline Nitrofurantoin & $174(71.3 \%)$ & $4(1.7 \%)$ & $66(27 \%)$ \\
\hline Norfloxacin & $76(31 \%)$ & 0 & $168(69 \%)$ \\
\hline Piperacillin/Tazobactam & $215(88.1 \%)$ & 0 & $29(11.9 \%)$ \\
\hline Imipenem & $244(100 \%)$ & 0 & 0 \\
\hline
\end{tabular}


Table.4 Various studies showing the prevalence of ESBL producing E. coli isolated from UTI

\begin{tabular}{|l|l|l|l|}
\hline S. No. & Studies & Year & Prevalence \\
\hline 1 & Datta P et al., $^{12}$ & 2014 & $21.4 \%$ \\
\hline 2 & Dugal S et al., $^{13}$ & 2013 & $24.4 \%$ \\
\hline 3 & DMBT Dissanayake et al., $^{14}$ & 2012 & $29 \%$ \\
\hline 4 & Chaudhary NK et al., ${ }^{15}$ & 2013 & $54.5 \%$ \\
\hline 5 & Mahesh E et al., $^{16}$ & 2010 & $56.2 \%$ \\
\hline 6 & Singh N et al., & 2016 & $82.6 \%$ \\
\hline $\mathbf{7}$ & Present Study & $\mathbf{2 0 1 7}$ & $\mathbf{6 1 \%}$ \\
\hline
\end{tabular}

\section{Graph 1: ESBL producers among clinical isolates}

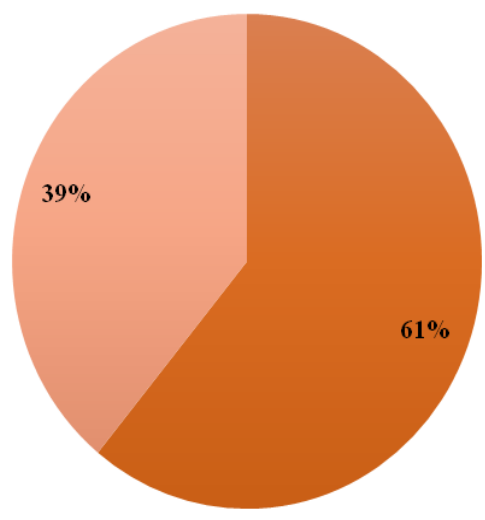

- ESBL producers

Non ESBL producers

\section{Graph 2: Gender Distribution}

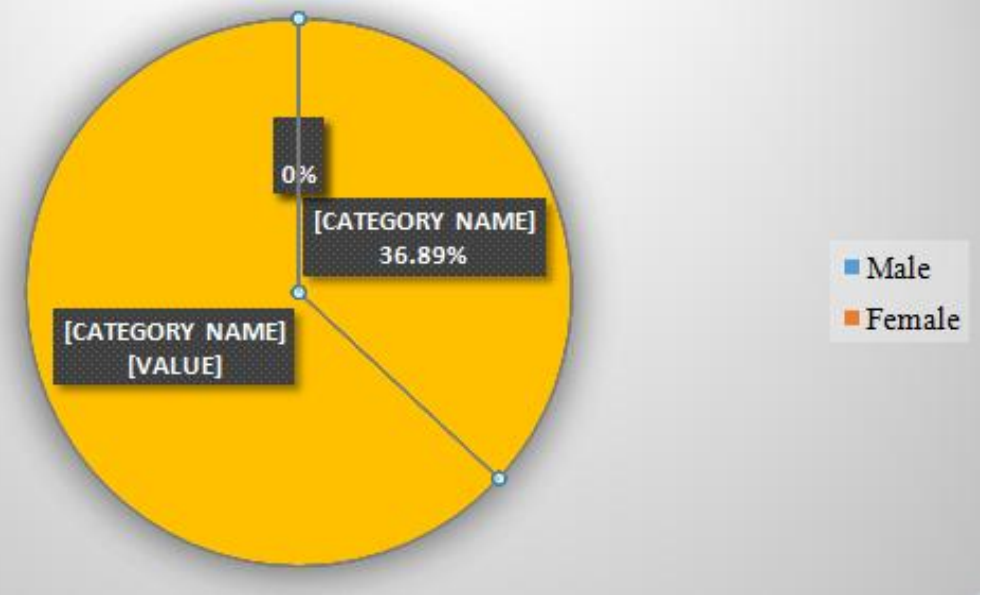




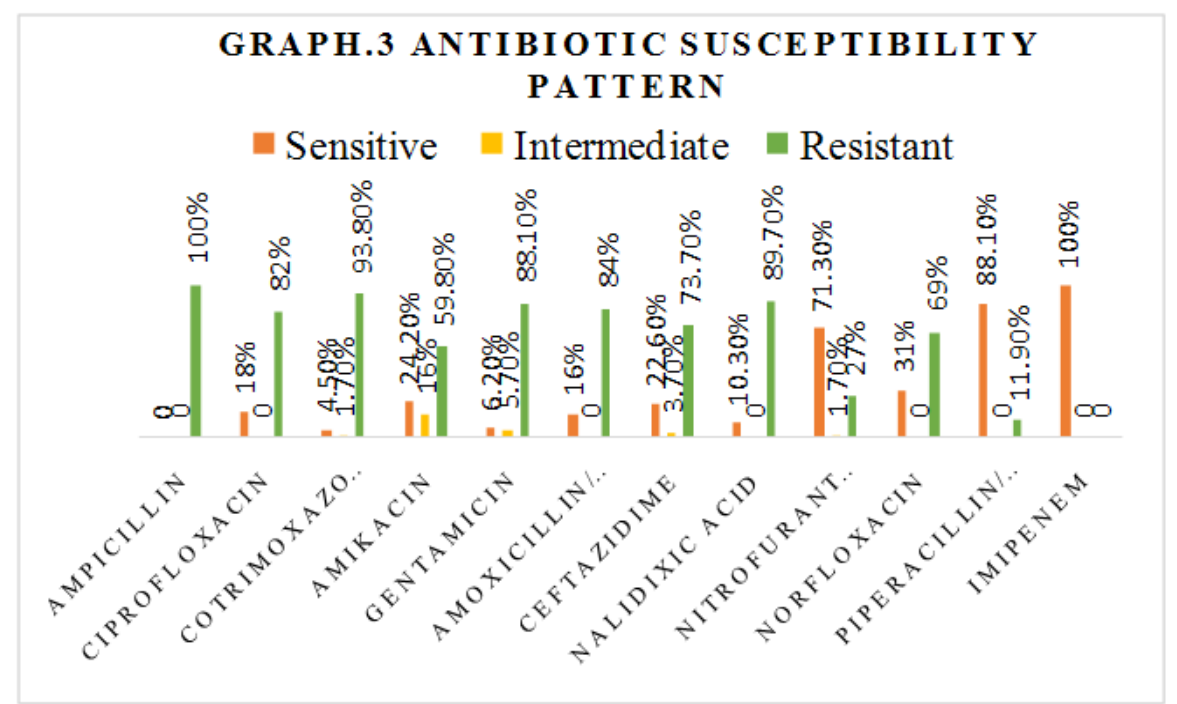

In the present study, we used phenotypic confirmation test (PCT) for detection of ESBL producer. PCT is technically much simpler and inexpensive compared to Double Disk Synergy Test (DDST). The interpretation is straight forward. Assuming that a laboratory is currently testing the sensitivity for ceftazidime and cefotaxime with the disk diffusion tests, only two disks are required to be added to the sensitivity plate to perform a PCT. This would screen all gram negative organisms in the laboratory for ESBL production (Selvakumar et al., 2007).

Ampicillin resistance among ESBL producing E. coli was found to be $100 \%$ which is similar to the finding of Behroozi et al., (2010) $(100 \%)$. Ciprofloxacin resistance was found to be $82 \%$ which is in correlation to the finding of Shafaq et al., (2011) (85\%). Cotrimoxazole resistance was found to be $93.8 \%$ which is higher than the findings of Chaudhary et al., (2013) (78.8\%) and Behroozi et al., (2010) (80\%). Amikacin resistance was $59.8 \%$ which is in correlation with the finding of Behroozi et al., (54\%). Gentamicin resistance was found to be $88 \%$ which is higher than the findings of Behroozi et al., (50\%), Chaudhary et al., (50.9\%) and Shafaq et al., (60\%). Amoxicillin/Clavulanate resistance was found to be $84 \%$ which is in correlation with Shafaq et al., (2011) (85\%) and Dutta et al., (2014) (88.5\%). Ceftazidime resistance was found to be $73.7 \%$ which is in between Daryl et al., (2012) (69\%) and Behroozi et al., (2010) (85\%). Nalidixic acid resistance was found to be $89.7 \%$ which is similar to Behroozi et al., (85\%). Nitrofurantoin resistance was $27 \%$ which is in between Behroozi et al., (20\%) and Chaudhary et al., (2013) (38.8\%). Piperacillin/ Tazobactam resistance was found to be $11.9 \%$ which is similar to the findings of Daryl et al., (2014) (16\%). Imipenem sensitivity was found to be $100 \%$ which is similar to the findings of Daryl et al., (2014) (100\%) and Shafaq et al., (2011) $(100 \%)$.

The present study demonstrates that some ESBL producing isolates show false susceptibility to third generation cephalosporin in in-vitro testing. Therefore, we recommend that detection of ESBL should be undertaken before starting UTI treatment.

In conclusion, the present study found $61 \%$ ESBL producing E. coli isolate in UTI. Most of the ESBL producing E. coli isolates were multidrug resistant making available 
therapeutic choices limited. Our study also demonstrates the importance of regular review of empirical antibiotic therapy for UTI in view of the evolving resistance of ESBL producing $E$. coli to commonly used agents.

Clinicians must depend on more laboratory guidance, while laboratories must provide resistance pattern data for optimal patient management more accurately. Additionally, robust antimicrobial stewardship and strengthened infection control measures are required to prevent the spread and reduce the emergence of antibiotic resistance.

\section{Acknowledgement}

We would like to thank our patients to agree for giving the consent and our family members for their support.

\section{References}

Baird, D. 2014. Staphylococcus: Clusterforming gram positive cocci. In: Collee JG, Fraser AG, Marmion BP, Simmons A, editors. Mackie and McCartney. Practical medical microbiology. 14th edition; Edinburg: Churchill Livingstone, 245-261.

Behrooozi, A., Rahbar, M., Yousefi, J.V. 2010. Frequency of extended spectrum beta lactamase (ESBLs) producing Escherichia coli and Klebsiella pneumoniae isolated from urine in an Iranian 1000 bed tertiary care hospital 4(9): 881-884.

Chaudhary, N.K., Murthy, S.M. 2013. Extended expectrum betalactamases in uropathogen. Asian J. Pharmaceutical and Clin. Res., 6(3): 207-210.

Cheesbrough, M. 1989. Medical Laboratory Manual for Tropical Countries, Vol II, Microbiology. Cambridge, Great Britain. pp 248-263.
Clinical and Laboratory Standards Institute. 2016. Performance Standards for Antimicrobial Susceptibility Testing; Twenty-six Informational Supplement. CLSI document M100-S26. Clinical and Laboratory Standards Institute, 940 West Valley Road, Suite 1400, Wayne, Pennsylvania 19087 USA.

Daryl, J.H., Christine, L., Lindsay, E.N., et al. 2012. Antimicrobial susceptibility of Enterobacteriaceae, including molecular characterization of extended spectrum beta lactamase producing species, in urinary tract isolates from hospitalized patients in North America and Europe: results from the SMART study 20092010. Diag. Microbiol. Infect. Dis., 74: 62-67.

Datta, P., Gupta, V., Sidhu, S., Chander, J. 2014. Community Urinary Tract Infection due to ESBL producing $E$. coli: epidemiology and susceptibility to oral antimicrobials including Mecillinam. Nepal J. Med. Sci., 3(1): 57.

Dissanayake, D.M.B.T., Fernando, S.S.N, Chandrasiri, N.S. 2012. The distribution and characteristics of ExtendedSpectrum $\quad \beta$-Lactamase $\quad$ (ESBL) producing Escherichia coli and Klebsiella species among urinary isolates in a tertiary care hospital. Sri Lanka J. Infect. Dis., 2(2): 30-36.

Dugal, S., Purohit, H. 2013. Antimicrobial susceptibility profile and detection of extended spectrum beta-lactamase production by gram negative uropathogens. Int. J. Pharmacy and Pharmaceutical Sci., 5(4): 434-438.

Foxman, B. 2002. Epidemiology of urinary tract infections: incidence, morbidity, and economic costs. Am. J. Med., 14(Suppl 1A): 5S-13S.

Kariuki, S., Revathi, G., Corkill, J., et al. 2001. Escherichia coli from commonly acquired urinary tract infections 
resistant to fluoroquinolones and extended spectrum betalactams. J. infect. Developing count, 1: 257-262.

Kumar, M.S., Lakshmi, V., Rajagopalan, R. 2006. Occurrence of extended spectrum beta-lactamases among

Enterobacteriaceae spp. isolated at a tertiary care institute. Indian J. Med. Microbiol., 24: 208-11.

Mahesh, E., Ramesh, D., Indumathi, V.A., et al. 2010. Risk Factors for Community Acquired Urinary Tract Infection caused by ESBL-producing Bacteria. JIACM, 11: 271-6.

Nathisuwan, S., Burgass, D.S., LewisII, J.S. 2001. ESBLs: Epidemiology, Detection and Treatment. Pharmacothera., 21(8): 920-928.

Rajan, S., Prabavathy, J. 2012. Antibiotic Sensitivity and Phenotypic Detection Of ESBL producing E. coli Strains Causing Urinary Tract Infection In a Community Hospital, Chennai, Tamil Nadu, India.. Webmed Central Pharmaceutical Sci., 3(11): WMC003840

Rawat, D., Nair, D. 2010. Extended-spectrum $\beta$-lactamases in Gram Negative Bacteria. J. Glob. Infect. Dis., 2(3): 26374.

Sasirekha, B. 2013. Prevalence of ESBL, AmpC $\beta$ - lactamases and MRSA Among uropathogens and its antibiogram. EXCLI J., 12: 81-88.
Selvakumar, B.N., Jasmine, R. 2007. Antibiotic susceptibility of ESBLproducing urinary isolates at a tertiary care hospital in Tiruchirappalli, South India. J. Med. Sci., 7: 443-6.

Shafaq, A.H., Jamal, S.A. and Mustafa, K. 2011. Occurrence of multidrug resistant and ESBL producing E. coli causing urinary tract infections. J. Basic and Appl. Sci., 7(1): 39-43.

Singh, N., Pattnaik, D., Neogi, D.K., Jena, J., Mallick, B. 2016. Prevalence of ESBL in Escherichia coli Isolates among ICU Patients in a Tertiary Care Hospital. $J$. Clin. Diag. Res., 10(9): 19-22.

Stamm, W.E. and Norrby, S.R. 2001. Urinary tract infections: disease panorama and challenges. J. Infect. Dis.,; 183, Suppl. 1: S1-S4.

Suganya, A., Jegadeesh kumar, D., Ravi, D. 2014. Evaluation of antimicrobial activity of Solanumxanthocarpum against Betalactamase and Biofilm producing microorganisms. Int. J. Novel Trends in Pharmaceutical Sci., 4(6): 188-193.

Sulochana Somasundaram, Gowthami, K.R., Helen, A., Srilekha, P. and Sivanandam, M. 2013. Detection and molecular characterization of extended spectrum of beta lactamase (ESBL) producing Escherichia coli. Int. J. Curr. Microbiol. Appl. Sci., 2(8): 196-205.

\section{How to cite this article:}

Ravindranath Gangane and Javeria Firdous. 2017. Isolation and Antibiotic Sensitivity Pattern of Extended Spectrum Beta Lactamases (ESBL) Producing Escherichia coli Isolated from Urinary Tract Infection. Int.J.Curr.Microbiol.App.Sci. 6(6): 279-286. doi: https://doi.org/10.20546/ijcmas.2017.606.034 